\title{
Traversal of a Polarized Epithelium by Pathogenic Neisseriae: Facilitation by Type IV Pili and Maintenance of Epithelial Barrier Function
}

\author{
Alexey J. Merz, Dean B. Rifenbery, Cindy Grove Arvidson, \\ and Magdalene So \\ Department of Molecular Microbiology and Immunology, Oregon \\ Health Sciences University, Portland, Oregon, U.S.A.
}

\begin{abstract}
Background: Gonococci (GC) and meningococci (MC) are gram-negative bacterial pathogens that infect human mucosal epithelia. We would like to understand the functions of specific bacterial components at each stage of mucosal colonization: adhesion, cell invasion, and traversal into subepithelial tissues. As no animal model of mucosal colonization by GC or MC is available, increasingly sophisticated in vitro approaches have been used to address these issues.

Materials and Methods: We adapted the polarized $\mathrm{T}_{84}$ human epithelial cell system to study GC and MC colonization. Epithelial barrier function was monitored by permeability to soluble tracers and with electrical resistance measurements. Polarized cells were used to assay bacterial traversal of the monolayers, and cells grown on plastic were used to assay adhesion and cell invasion.

Results: All pathogenic Neisseriae examined traversed
\end{abstract}

the monolayers. The traversal times were species specific and identical to times established previously in organ culture studies. In contrast to experiments with some enteric pathogens, transmigration by GC and MC was not accompanied by disruption of the epithelial barrier. GC mutants lacking type IV pili were compromised in adhesion, invasion, and traversal of $\mathrm{T}_{84}$ cells.

Conclusions: Experiments with polarized $\mathrm{T}_{\mathbf{8 4}}$ cells mimic key features of organ culture infections and reveal additional aspects of neisserial infection. Epithelial barrier function can be retained during bacterial traversal. Experiments with a nonpiliated GC mutant and its wildtype parent indicated an unexpected role for pili in cell invasion. Our results are consistent with the hypothesis that bacterial adhesion, invasion, or both are rate-limiting for traversal across the epithelium.

\section{INTRODUCTION}

The genus Neisseria comprises several gram-negative diplococci that colonize human mucosal tissues $(1,2)$. In most cases the relationship between host and bacterium is commensal but some species can pose serious health risks. Neisseria gonorrhoeae (GC) commonly colonize the mucosa and submucosal stroma of the conjunctiva, throat, urogenital tract, and rectum. Infection at the latter sites may be a mechanical cofactor facilitating the transmission of human immunodeficiency virus $(3,4)$. Neisseria meningi-

Address correspondence and reprint requests to: Alexey J. Merz, Department of Molecular Microbiology and Immunology, Oregon Health Sciences University, L220, 3181

S.W. Sam Jackson Park Road, Portland, OR 97201, U.S.A. tidis (MC) colonize the nasopharynx, usually without causing disease. In some cases, however, MC may traverse the mucosal barrier and enter the blood, leading to septicemia. Furthermore, MC may cross the blood-brain barrier, resulting in meningitis.

Humans are the only natural host for GC and MC. As a result, no animal model of Neisseriamucosa interactions is presently available. However, segments of human oviduct (fallopian tube organ culture [FTOC]) and nasopharynx (nasopharyngeal organ culture [NPOC]) inoculated ex situ with GC and MC have yielded morphological descriptions of the initial steps of infection. Certain features are common to both GC and MC infection $(5,6)$. (i) Bacteria selectively adhere to 
secretory (nonciliated) epithelial cells, probably using pili. (ii) Bacteria intimately contact the host cell surface (close association). (iii) The region of contact enlarges until diplococci are surrounded by host plasma membrane. A fusion event is presumed to seal off the nascent vesicle. The engulfment process closely resembles classical "zippering" phagocytosis (7). In tissue culture cells, bacterial entry is blocked by both microfilament-disrupting drugs and inhibitors of bacterial protein synthesis, implicating both host and parasite as active participants $(8,9)$. (iv) GC survive and multiply inside epithelial cells. (v) GC are observed to traverse the length of the infected cell, and by $1-2$ days after infection GC or MC is observed in the stromal matrix $(5,6)$.

The available infection models have advantages and limitations. Human volunteer studies have provided data implicating pili and opacity (Opa) proteins in colonization (10). But volunteer studies and organ culture are expensive and limited by the availability of volunteers or tissue specimens, so they cannot be used to compare many different bacterial strains. Cultured human cells grown on plastic have been used to dissect the function of a number of GC and MC surface components (11-16). However, cells grown on plastic do not replicate the polarized architecture of epithelial and endothelial cells in situ and are therefore less useful for studying events after the early colonization steps. Moreover, none of the above models permits the isolation of viable bacteria from subepithelial spaces, and none of the in vitro systems facilitates studies of the mucosal inflammatory response, a hallmark of GC infection (1).

Many of these limitations can be overcome by culturing epithelial cells on permeable membrane supports which permit cells to feed from their basolateral surface and to polarize as in native tissue $(17,18)$. Both "serosal" and "mucosal" surfaces are experimentally accessible, permitting studies of transcytosis and protein trafficking $(19,20)$, barrier physiology $(21,22)$, bacterial infection $(23,24)$, and leukocyte diapedesis $(25,26)$. A recent report (27) describes a system that incorporates both an epithelial (HEC-1-B) and an endothelial layer (HMEC-1) to study early MC pathogenesis.

We adapted the polarized $\mathrm{T}_{84}$ colonic epithelial cell model to study Neisseria-mucosa interactions. GC and $M C$ traversed $\mathrm{T}_{84}$ monolayers in distinct time courses identical to those observed in organ culture studies. In contrast to other studies with MC and with pathogenic Escherichia coli and Salmonella $(23,24,27-29)$, traversal of ep- ithelial monolayers by GC and MC was not accompanied by disruption of the epithelial barrier. In addition, we show that pili facilitate not only GC adhesion but invasion and traversal of $T_{84}$ monolayers. These latter features have not been reported previously.

\section{MATERIALS AND METHODS}

\section{Bacterial Strains}

GC MS11A has two pilin expression loci, pilE1 and pilE2 (30). To create a nonreverting pilE mutant MS11C3, a strain deleted in pilE2 (31) was used as the parent in our constructions. To delete pilEl a $2.1 \mathrm{~kb}$ Bsu36I fragment from pNG1711 (30) was replaced with a 1.2 kb erythromycin resistance $\left(\mathrm{Erm}^{\mathrm{r}}\right)$ cassette from pATl10 (32). This plasmid was linearized and used to transform MS11C3 (pilE2). A nonpiliated, $\mathrm{Erm}^{\mathrm{r}}$ transformant was isolated and named MS11306. PCR and Southern blotting verified that pilEl was deleted (not shown). MS11-306 is nonpiliated, and MS11A, F62, and MC8013.6 are piliated as assessed by immunoblot with monoclonal antibody (mAb) 10H5.1.1 (14) and by colony morphology or electron microscopy. MS $11 \mathrm{~A}$ and MS11-306 are $\mathrm{Opa}^{-}$by immunoblot with $\mathrm{mAb} 4 \mathrm{~B} 12$, generously provided by $\mathrm{M}$. Blake (not shown). MC 249 and 250 are uncharacterized with respect to piliation and Opa/Opc production. For infection experiments bacterial inocula were grown for 18 hours on GCB agar with nutritional supplements at $37^{\circ} \mathrm{C}, 5 \% \mathrm{CO}_{2}$ as described (33). Table 1 outlines the bacterial strains used.

\section{Tissue Culture}

$\mathrm{T}_{84}$ cells from the American Type Culture Collection were grown between passages 55 and 70 in a 1:1 mix of Dulbecco's modified Eagle's medium (DMEM) and Ham's F12 (Whittaker, Walkersville, MD, U.S.A.) with $5 \%$ heat-inactivated fetal bovine serum (FBS; Hyclone, Logan, UT, U.S.A.; or Gibco-BRL, Gaithersburg, MD, U.S.A.). For infection experiments this culture medium was supplemented with human transferrin except as noted $(>90 \%$ iron-saturated; Boeringer-Mannheim, Indianapolis, IN, U.S.A.) at $5 \mu \mathrm{g} / \mathrm{ml}$. Cells were harvested and plated $\left(10^{6}\right.$ cells $\left.\mathrm{cm}^{-2}\right)$ on Transwell filters (\#3415; Costar, Cambridge, MA, U.S.A.) coated with rat tail collagen I (Collaborative Biotech Bedford, MA, U.S.A.) as described 
TABLE 1. Bacterial strains used in these studies

\begin{tabular}{|c|c|c|}
\hline \multicolumn{2}{|l|}{ Strain } & Reference \\
\hline \multicolumn{3}{|c|}{ N. gonorrhoeae } \\
\hline MSIIA & $\mathrm{P}^{+}, \mathrm{Opa}^{-}$ & 31 \\
\hline MS11-306 & $\triangle$ pilE1::Erm, $\triangle$ pilE2, $\mathrm{Op}^{-}$ & This article \\
\hline MS11A-14. & $.1 \mathrm{P}^{+}, \mathrm{Opa}^{-}$, opaH::Cat & 33 \\
\hline F62 & & $33 a$. \\
\hline \multicolumn{3}{|c|}{ N. meningitidis } \\
\hline 249,250 & $\begin{array}{l}\text { Blood and cerebrospinal fluid isolates from a single patient; } \\
\text { encapsulated; serogroup C }\end{array}$ & This article \\
\hline 8013.6 & $\begin{array}{l}\mathrm{P}^{+} \text {; highly adhesive to cultured epithelial and endothelial } \\
\text { cells; encapsulated; serogroup } \mathrm{C}\end{array}$ & 14 \\
\hline \multicolumn{3}{|l|}{ Others } \\
\hline \multicolumn{3}{|c|}{ N. perflava NRL-30015 } \\
\hline N. sicca & $N R L-30016$ & Dr. Joan Knapp, CDC \\
\hline
\end{tabular}

$(21,26)$. These monolayers localized human transferrin receptor predominantly basolaterally and exhibited classical junctional complexes and dense microvilli when stained for filamentous actin (not shown). Monolayer formation was routinely assayed by measuring electrical resistance with the ECL device (Millipore, Bedford, MA, U.S.A.). In our hands monolayers took 7-14 days to exceed $600 \Omega \mathrm{cm}^{2}$.

\section{Adhesion and Invasion Assays}

Adhesion and invasion were scored by washing, lysing, and plating of the infected cells before or after killing of extracellular bacteria with gentamicin. Assays were done as before $(8,33)$, except that DMEM-F12 supplemented as above was used instead of DMEM, and GC broth containing $0.5 \%(\mathrm{w} / \mathrm{v})$ saponin was used to lyse the epithelial cells. To decouple invasion efficiency from differences in bacterial adherence, invasion indices were derived by dividing the number of internalized (gentamicin-resistant [GR]) colonyforming units (CFU) by the number of adherent (cell-associated [CA]) CFU.

\section{Monolayer Traversal Experiments}

Polarized $\mathrm{T}_{84}$ monolayers with resistances $>600 \Omega \mathrm{cm}^{2}$ were rinsed with medium, placed into fresh 24-well plates containing $0.5 \mathrm{ml}$ of the invasion medium described above, and $100 \mu \mathrm{l}$ of invasion medium was added to the apical well. Bacteria were suspended in DMEM-F12 at $10^{8} \mathrm{CFU} / \mathrm{ml}, 10$ $\mu \mathrm{l}$ of which was added to the apical medium. Infected cells were incubated at $37^{\circ} \mathrm{C}, 5 \% \mathrm{CO}_{2}$. At intervals filters were moved to new 24 -well plates containing fresh medium, and the old medium was plated on GCB agar with nutritional supplements.

To quantitate MC flux across monolayers the above protocol was followed, but at $12 \mathrm{hr}$ postinfection and at 1- or 2-hr intervals thereafter the basal medium was changed to DMEM-F1 2 with $5 \%$ FBS. The newly removed basal medium was diluted and plated to enumerate CFUs. At the end of the assay ( $24 \mathrm{hr}$ postinfection) each filter was assayed for transepithelial resistance to verify that barrier function was intact $\left(\geq 500 \Omega \mathrm{cm}^{2}\right)$. The time of traversal varied slightly with the batch of fetal bovine serum used, so each set of experiments shown was performed using a single lot of serum.

\section{Permeability Assay}

Monolayer permeability to $\left[{ }^{3} \mathrm{H}\right]$ mannitol was determined as described (34). Briefly, basal medium was removed and plated for CFU, filters were placed into new 24-well plates containing fresh basal medium, and the apical medium was replaced with $100 \mu \mathrm{l}$ of DMEM-F12 containing $\sim 10^{4} \mathrm{cpm}$ of $\left[{ }^{3} \mathrm{H}\right]$ mannitol (New England $\mathrm{Nu}-$ clear, Boston, MA, U.S.A.). Filters were incubated for $60 \mathrm{~min}$. At the end of this interval medium was removed from both chambers and counted with a liquid scintillation detector. Data are expressed as described (34). 
TABLE 2. Adhesion and invasion of $\mathbf{T}_{\mathbf{8 4}}$ cells grown on plastic by Neisseria strains, after a 7-hr infection

\begin{tabular}{lccc}
\hline & Cell-Associated CFU (CA) & Gentamicin $^{\mathbf{r}}$ CFU (GR) & Invasion Index (GR/CA) \\
\hline GC MS11A & $1.19(0.48) \times 10^{6}$ & $1.25(0.87) \times 10^{3}$ & $1.1 \times 10^{-3}$ \\
GC MS11 A-14.1 & $1.33(0.28) \times 10^{6}$ & $5.53(1.2) \times 10^{2}$ & $3.5 \times 10^{-4}$ \\
N. sicca & $1.23(0.18) \times 10^{7}$ & $4.80(1.3) \times 10^{2}$ & $3.9 \times 10^{-5}$ \\
\hline
\end{tabular}

Invasion was measured with a gentamicin protection assay (see Materials and Methods). Mean and SD of three to six replicates from a representative experiment are shown.

\section{Fluorescence Microscopy}

Actin staining was performed by fixing filters for 30 min in Zamboni's fixative (PAPF) (35) at room temperature followed by permeablization in phosphate-buffered saline (PBS) with $0.1 \%$ goat serum, $0.01 \%$ azide, and $0.02 \%$ saponin. Actin was stained for $1 \mathrm{hr}$ with BODIPY 581/ 591-phalloidin (Molecular Probes, Eugene, OR, U.S.A.) diluted 1:200 from methanol stock made as recommended. Filters were rinsed extensively and mounted in glycerol. Images were acquired with a Leica inverted laser-scanning confocal microscope.

\section{Statistics}

Invasion indices and raw CFU counts log-transformed to normalize variances were compared using Student's two-tailed, paired $t$ test where appropriate. Results from the traversal time course experiments were evaluated using the Mann-Whitney $U$ test when sample sizes were equal or the log likelihood ratio $(G)$ test when sample sizes were unequal. Calculations were set up as recommended (36) and done using StatView 4.0 software for the Macintosh (Abacus products, Berkeley, CA, U.S.A.).

\section{RESULTS}

\section{$T_{84}$ Cells Support Adhesion and Invasion of Pathogenic Neisseria}

The barrier (gate) function of epithelial cells is critical to tissue function in vivo (22). In vitro, it is a stringent indicator of monolayer integrity and epithelial polarity, and can be assayed by impermeability to small tracers or by transmonolayer electrical resistance. Only a few human epithelial cell lines have been successfully grown as polarized monolayers with high electrical resistance. For example HEC-1-B, a human endometrial carcinoma line commonly used to study GC and MC infection, polarized morphologically when grown on Transwell filters coated with collagen I or Matrigel but in our hands did not attain high electrical resistance (data not shown). The $\mathrm{T}_{84}$ cell line, derived from a human colon carcinoma, is known to form polarized monolayers with robust barrier function (21). Additionally, colorectal epithelia are sites of clinical GC infection. We therefore asked whether $\mathrm{T}_{84}$ cells are appropriate for studying neisserial-epithelial interactions.

Adherence and invasion indices of GC for $\mathrm{T}_{84}$ cells grown on plastic were compared with those for HEC-1-B cells. Results indicated that GC MSIlA (piliated, $\mathrm{Opa}^{-}$) adhered to and invaded $\mathrm{T}_{84}$ cells at the same frequency as HEC-1-B cells $(8,9,33)$. For example, at $7 \mathrm{hr}$ postinfection strain MS11A had an invasion index of $\sim 10^{-3}$ in both HEC-1-B (33) and $T_{84}$ cells (Table 2). Furthermore, MS11A-14.1, an opaH mutant previously shown to be reduced 4.3 -fold in its ability to invade HEC-1-B cells (33), was similarly reduced (3- to 6-fold) in its ability to invade $\mathrm{T}_{84}$ cells (Table 2). These results indicated that $\mathrm{T}_{84}$ cells would be a useful model for GC-epithelial cell interactions. Similar molecular mechanisms govern many GC and MC interactions with host cells. For example, type IV pili, PilC, and Opa are involved in adhesion and invasion $(1,2)$. Therefore, the $\mathrm{T}_{84}$ model may be appropriate for studies of MC-host cell interactions as well.

\section{MC and GC Traverse $T_{84}$ Cells in Species- Specific Time Courses Identical to Those Established in Organ Culture Studies}

In FTOC and NPOC studies, GC and MC entered epithelial cells at the apical region, traversed to 
the basal region, and egressed into the stromal matrix $(5,6)$. To determine whether Neisseria spp. can traverse polarized $T_{84}$ cells, we infected monolayers plated on collagen-coated Transwell filters. Only monolayers exceeding $600 \Omega \mathrm{cm}^{2}$ were used in this study; most were $>1000 \Omega$ $\mathrm{cm}^{2}$. Polarized $\mathrm{T}_{84}$ monolayers were infected with three clinical isolates of $\mathrm{MC}$, one clinical isolate and two laboratory strains of GC, and a nonpathogenic Neisseria strain (see Materials and Methods). At 12-hr intervals, the medium from the basal well of each filter unit was plated to detect colony-forming units (CFUs). Each strain was assayed in several independent experiments with 3-12 filters per strain per assay.

Results indicate that Neisseria strains traversed $\mathrm{T}_{84}$ monolayers with different kinetics (Fig. 1A). Most MC-infected filters yielded CFUs in the basal medium at 12-24 hr postinfection, although several filters yielded MC in the basal medium by $12 \mathrm{hr}$ postinfection. In contrast, most GC-infected filters yielded CFU in the basal compartment significantly later, at $36-48 \mathrm{hr}$ postinfection $(p<0.001 ; \log$ likelihood ratios test). The traversal times observed for MC and GC in these assays are strikingly similar to times reported for FTOC and NPOC experiments: $>18 \mathrm{hr}$ for MC and $\sim 40 \mathrm{hr}$ for GC $(37,38)$.

At the times assayed, Neisseria perflava was only rarely detected in the basal chamber (Fig. 1A). E. coli $\mathrm{HB} 101$ traversed $\mathrm{T}_{\mathbf{8 4}}$ monolayers and reached the basal compartment within 12 $\mathrm{hr}$, however this was accompanied by a nearly complete loss of epithelial barrier function (data not shown), consistent with experiments using MDCK cells (23).

Assays to determine the number of bacteria traversing the monolayers were also performed. Monolayers were infected with $\mathrm{MC}$ for a defined period ( $12 \mathrm{hr}$ for $\mathrm{MC} ; 30 \mathrm{hr}$ for GC) then moved into fresh basal medium lacking serum and transferrin. The basal medium from the previous interval was plated for CFUs at 1- or 2-hour intervals. In medium without supplements, GC and MC grow slowly (data not shown). Thus, our estimates of basal well CFUs should closely approximate the actual number of bacteria exiting the monolayer.

Results from an experiment with MC 8013.6 are shown (Fig. 1B). By $20 \mathrm{hr}$ postinfection, in many wells $10^{2}-10^{4} \mathrm{CFU} / \mathrm{hr}$ were entering the basal compartment. At this time 1-2 $\times 10^{7}$ CFU were present in the apical compartment. Similar results were obtained with GC MSI IA at $\sim 40 \mathrm{hr}$ postinfection (not shown).

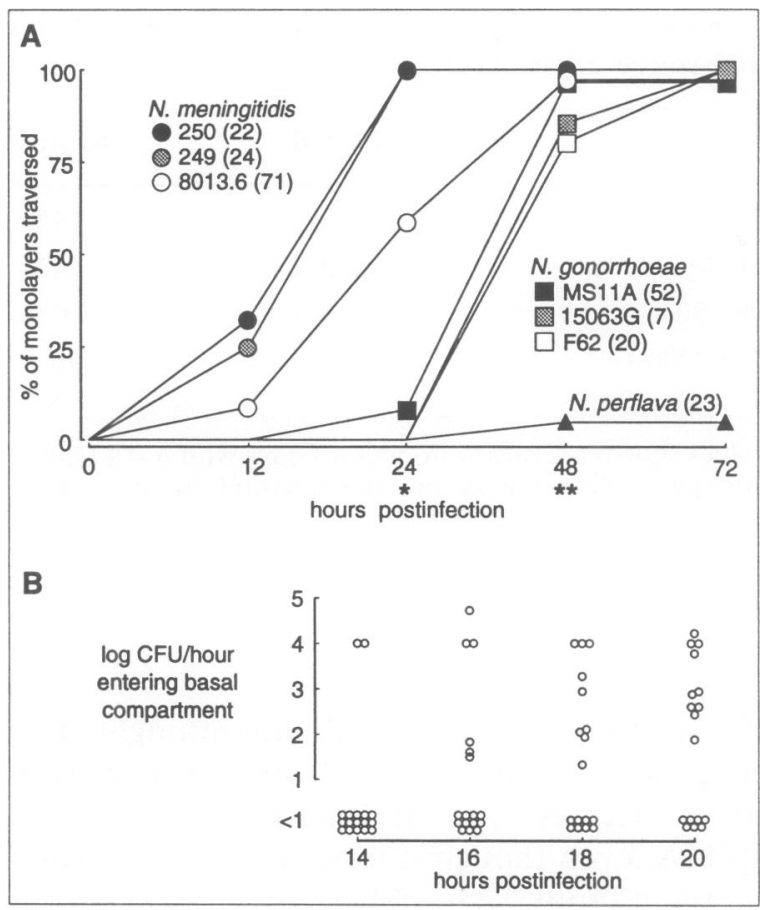

FIG. 1. Traversal across $\mathbf{T}_{\mathbf{8 4}}$ monolayers by different Neisseria strains.

(A) One million CFU of each of the indicated strains were inoculated into the apical wells of polarized $\mathrm{T}_{84}$ monolayers. At 6-, 12-, or 24-hr intervals, the filters were moved to wells with fresh medium and the medium from the old wells was plated on supplemented GCB agar. Points show the percentage of wells for a given strain that had detectable CFU in the lower chamber during each interval. The numbers in parentheses after each strain designation show the number of monolayers assayed. *The time taken by MC to traverse NPOC epithelia (3). **The time taken by GC to traverse FTOC epithelia (4). (B) Flux of MC 8013.6 into the basal compartment. At $12 \mathrm{hr}$ postinfection medium in the basal wells was replaced with unsupplemented DMEM-F12 to limit the rate of bacterial growth. At intervals filter units were moved to fresh basal medium and the old medium was plated. Twelve additional monolayers were infected with $N$. perflava and did not yield CFU during any of the intervals. A representative experiment is shown.

\section{Barrier Function of Infected Monolayers}

To determine whether the passage of bacteria across $\mathrm{T}_{\mathbf{8 4}}$ monolayers affects epithelial barrier function, the electrical resistances of GC, MC, and $N$. perflava-infected filters were measured (Table 3). Results indicate that traversal could occur without large decreases in barrier function. For example, at $48 \mathrm{hr}$ postinfection GC MS11Ainfected monolayers were decreased an average of $15 \%$ from starting values. At this time, bacterial traversal had already occurred in over $90 \%$ 
TABLE 3. Changes in transepithelial electrical resistance of infected $T_{84}$ monolayers

\begin{tabular}{lcc}
\hline & 24 hpi & $\mathbf{4 8 ~ h p i}$ \\
\hline GC MS11A & nd & $-15 \%$ \\
GC MS11-306 & nd & $-54 \%$ \\
MC 8013.6 & $-33 \%$ & $-70 \%$ \\
N. perflava & $+11 \%$ & nd \\
\hline
\end{tabular}

Values shown are means from 2-4 assays with 6-18 replicates per strain per assay. hpi, hours postinfection; nd, not done.

of the monolayers assayed. Interestingly, the nonpiliated mutant GC MS1 1-306 always caused larger decreases in resistance than piliated MS11A, even though it traversed the monolayers significantly later (Table 3; see below). This result indicates that a modest decrease in barrier function is not sufficient to permit bacterial traversal. At $24 \mathrm{hr}$ postinfection MC $8013.6 \mathrm{de}$ creased resistances by an average of $33 \%$ from starting values. At this time, the values obtained for MC-infected monolayers with detectable basal CFU ranged from 540 to $1800 \Omega \mathrm{cm}^{2}$. By 48 $\mathrm{hr}$ postinfection, resistances of $\mathrm{MC}$-infected monolayers declined by $\sim 70 \%$ from starting values. This decrease is not surprising, as the basal medium of these filters usually contained over $10^{8} \mathrm{CFUs} / \mathrm{ml}$ at this time, contained by-products of bacterial growth, and was acidic $(\mathrm{pH} \leq 6.5)$ compared with the controls.

To confirm the electrical resistance data, infected monolayers were tested for permeability to a small soluble tracer, $\left[{ }^{3} \mathrm{H}\right]$ mannitol. Individual filters were assayed for both bacterial transit and permeability. As a positive control for permeability, barrier function in some uninfected monolayers was abolished by treatment with EDTA (10 mM, $15 \mathrm{~min}$ ) prior to the assay. Results (Fig. 2) show that the MC 8013.6-infected monolayers had somewhat increased permeability compared with uninfected monolayers, although they were still several-fold less permeable than the EDTA-treated controls. Similar results (Fig. 2) were obtained for MC strains 249 and 250, and GC MS11A (not shown). Bacterial transit and epithelial permeability are not correlated: in many samples with CFUs present in the lower chamber, the barrier function was identical to uninfected controls. In other samples,

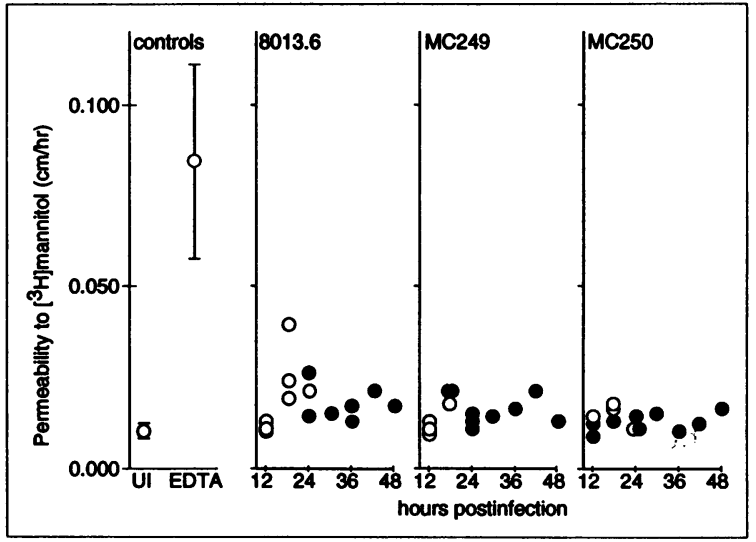

FIG. 2. Permeability to $\left[{ }^{3} \mathrm{H}\right]$ mannitol of individual infected monolayers

The far left column shows the mean \pm SD $(n=6)$ of control wells, either uninfected (UI) or uninfected but treated for $15 \mathrm{~min}$ with $10 \mathrm{mM}$ EDTA. Both positive and negative controls were done at different times after infection with similar results. The other columns show results for single wells infected with the indicated strains for different amounts of time. - samples in which bacteria had crossed the monolayer; $O$, samples in which no bacteria were detected in the basal medium. One of two independent experiments.

where permeability had increased, no bacterial transit was detected. We conclude that as GC and MC traverse the $\mathrm{T}_{84}$ monolayer, a functional epithelial barrier is maintained.

\section{Cytoarchitecture of Infected Monolayers}

To characterize further the effects of bacterial traversal on epithelial integrity, the arrangement of actin filaments in infected $\mathrm{T}_{84}$ monolayers was examined. Polarized monolayers were infected with MC 8013.6 for $40 \mathrm{hr}$, fixed, and stained. Confocal optical sections at and just beneath the apical surface revealed that both uninfected and infected cells were circumscribed by the apicolateral actin band associated with adherens and occludens junctional complexes (39). In the filters shown (Fig. 3), bacteria had traversed the monolayers at the time of fixation. Similar results were obtained for GC MS11A-infected monolayers (data not shown). These results provide further evidence for the maintenance of cell polarity and barrier function during and after bacterial traversal. In contrast, the apicolateral actin band in Salmonella-infected MDCK II cells was disrupted by $1 \mathrm{hr}$ postinfection (29). 

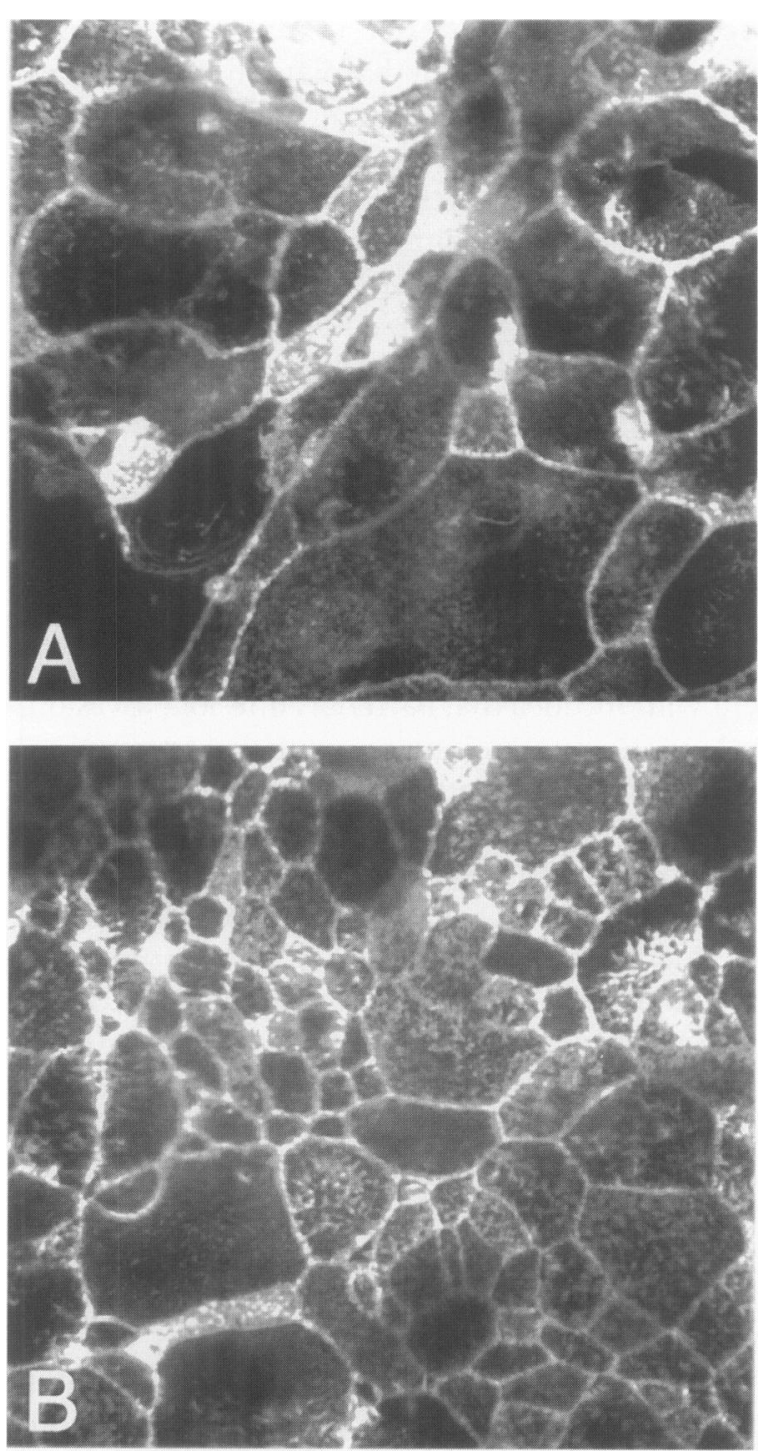

FIG. 3. Actin staining at $40 \mathrm{hr}$ in control $\mathrm{T}_{\mathbf{8 4}}$ cells (A) or in cells infected with MC8013.6 (B).

Confocal optical sections of apicolateral regions of the monolayers were made with a $40 \times$ objective. The photos show an area of $73 \times 73 \mu \mathrm{m}$. One of three experiments.

\section{Role of Pili in GC Interactions with $\mathbf{T}_{\mathbf{8 4}}$ Cells}

As GC type IV pili are thought to be essential for virulence in human volunteers $(40,41)$, we compared interactions of piliated GC MS11A with its isogenic nonpiliated derivative MS11-306 using $\mathrm{T}_{84}$ cells. In infections of $\mathrm{T}_{84}$ cells plated on plastic (Fig. 4A), pili increased GC adherence by $\sim 20$-fold. At 5, 7.5, and $10 \mathrm{hr}$ postinfection $50-$ $70 \%$ of MS11A were cell-associated (CA) compared with only $2-4 \%$ of MS11-306. Surprisingly, pili also promoted GC invasion of $\mathrm{T}_{84}$ cells.

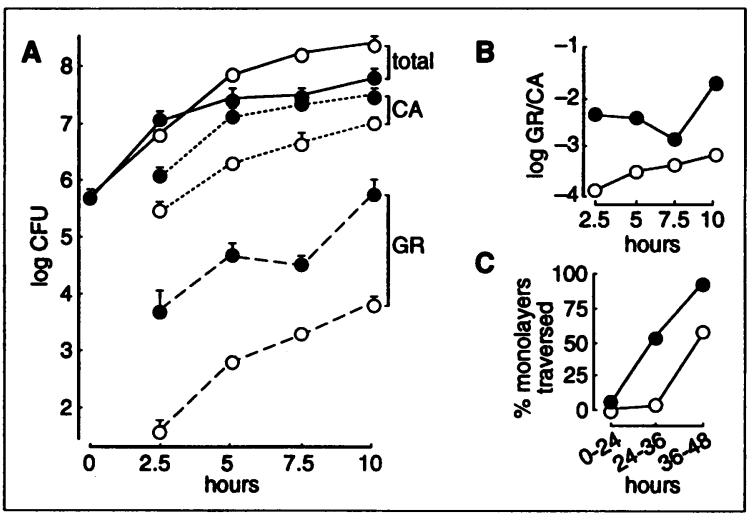

FIG. 4. Effects of pili on GC interactions with $\mathbf{T}_{\mathbf{8 4}}$ monolayers.

(A) Adherence and invasion of (O) MS1lA and (O) MS11-306 assayed with $T_{84}$ cells grown on plastic. CA, cell-associated; GR, gentamicin resistant. Each point shows mean + SEM of determinations from three or four independent experiments, each done at the least in triplicate. (B) Invasion indices. Invasion data from Panel A are shown normalized against adherence (intracellular CFU/cell-associated CFU). (C) Time course of traversal across $\mathrm{T}_{84}$ monolayers by the same strains. Data are presented as in Fig. 1.

At each time point 1-2 logs more gentamicinresistant (GR) MS11A were recovered than MS11-306 (mean difference, 42.4-fold; $p<$ $0.0001, t$ test) (Fig. 4A). This result cannot be explained solely by differences in adherence. If the invasion data are expressed as an index adjusted to compensate for adhesion (GR/CA), MS11A invaded $\mathrm{T}_{84}$ cells much more efficiently than MS11-306 (mean difference, 10.1-fold; $p=$ $0.0011, t$ test) (Fig. 4B). Piliation has been observed to promote $\mathrm{MC}$ and GC adhesion to cultured cells (Fig. 3A) $(14,15,42,43)$. This is the first report in which pili are shown to enhance GC invasion of cultured cells.

GC MS11A and MS11-306 were next compared for their ability to traverse polarized $\mathrm{T}_{84}$ monolayers (Fig. 4C). In these assays piliated MS11A appeared in the basal compartment about 12 hr earlier than MS11-306 ( $p<0.001$; Mann-Whitney two-tailed $U$ test). We emphasize that nonpiliated GC were at least as numerous in the apical chamber as piliated GC yet they crossed the monolayers later (Fig. 4C; data not shown). Thus, epithelial traversal is not simply a function of bacterial growth in the apical chamber. Together, these experiments provide evidence that pilus-mediated interactions with host cells, and possibly cell invasion, promote the traversal of GC across polarized $\mathrm{T}_{84}$ monolayers. 


\section{DISCUSSION}

The experiments reported here establish polarized $\mathrm{T}_{84}$ colonic carcinoma cells as a model for studies of epithelial infection by the pathogenic Neisseriae. Nonpolarized $\mathrm{T}_{84}$ cells grown on plastic are similar to the widely used HEC-1-B endometrial carcinoma cell line $(8,9,33)$ in their capacity to support GC adherence and invasion. The time courses of GC and MC transit across polarized monolayers are species specific and are identical to results obtained previously in organ culture studies $(5,6,37,38)$.

Transepithelial migration by pathogenic Neisseriae does not abolish the barrier function of polarized $\mathrm{T}_{84}$ monolayers. This conclusion is based on three types of experiments. First, monolayers which have been traversed by GC or MC are relatively impermeable to small soluble tracers (Fig. 2). Second, they have high electrical resistances, in excess of $500 \Omega \mathrm{cm}^{2}$. Resistances of $\sim 200 \Omega \mathrm{cm}^{2}$ are typical for polarized, uninfected monolayers of $\mathrm{CaCo} 2$ or MDCK II cells and are generally considered diagnostic of physiological barrier function (21). Third, apicolateral junctional complexes remain intact as judged by microfilament staining (Fig. 3 ). The small reductions in barrier function that were observed could be due to modulation of paracellular pathways, intracellular pathways, or both (22). The present experiments do not discriminate among these possibilities and should therefore provide conservative estimates of junctional integrity.

How can the maintenance of a substantial barrier in infected $\mathrm{T}_{84}$ monolayers be reconciled with organ culture data showing that GC and MC cause cytotoxicity and sloughing of some cells? It should be emphasized that in organ cultures, GC and MC selectively adhere to and invade nonciliated epithelial cells but that most of the evident toxicity occurs in ciliated cells $(5,6,37,38)$. This cell type-specific cytotoxicity is thought to be triggered by soluble bacterial components, particularly peptidoglycan and lipooligosaccharide, and to be mediated by the release of tumor necrosis factor $\alpha(1)$. Many tissues infected by GC (e.g., conjunctival, uterine and colorectal epithelia) do not contain ciliated cells, and severe epithelial effacement is not generally noted in clinical cases of Neisseria infection. $\mathrm{T}_{84}$ cells may therefore be viewed as a model for the population of nonciliated cells that are colonized in vivo.

It is possible that the cellular targets for neisserial toxins are localized basolaterally and that the robust barrier present in the $\mathrm{T}_{84}$ system may prevent soluble bacterial toxins in the apical chamber from reaching basolateral cell surfaces. This hypothesis is consistent with our observation that barrier function is largely retained even when very many bacteria are present in the apical chamber but is diminished after large numbers of bacteria accumulate in the basal chamber.

Pili are important for colonization of the urogenital tract in vivo and facilitate GC interactions with ex vivo FTOC cells $(1,2,5,40,41)$. Consistent with these results, our data show that pili strongly enhance GC adhesion, invasion, and traversal of polarized $\mathrm{T}_{84}$ monolayers. Likewise, piliated $\mathrm{MC}$ 8013 traverses $T_{84}$ monolayers more efficiently than an isogenic nonpiliated counterpart (X. Nassif, personal communication). These results are consistent with the idea that bacterial adhesion, invasion, or both are rate-limiting for epithelial traversal.

Similar to our results with $\mathrm{T}_{84}$ cells, Makino et al. (42) report that, with Chang conjunctival cells, piliated GC are more adherent than nonpiliated strains. In contrast, while piliation causes a 1 log increase in invasion efficiency with $\mathrm{T}_{84}$ cells, Makino et al. (42) report a 1-2 log decrease with Chang cells. When our GC strains are assayed with Chang cells, the invasion efficiency of the piliated strain is similarly decreased (A. Merz, unpublished data). Thus this difference is not due to the particular GC strains used. Moreover, with A431 cervical carcinoma cells an intermediate result is obtained: piliation strongly affects adhesion but not invasion efficiency (C. G. Arvidson, unpublished). The effect of piliation on GC invasion efficiency therefore depends strongly on the cells used, and ranges from a 1-2 log inhibition (Chang) to little or no effect (A431) to a 1 log enhancement $\left(\mathrm{T}_{84}\right)$. We suggest that host cell-specific differences in the effects of type IV pili on GC invasion reflect a previously unrecognized aspect of the in vivo GC-host interaction. This possibility is being investigated.

Several approaches have been used to study Neisseria-host interactions in vitro. Cell culture has been used to identify numerous bacterial components as adherence and invasion factors. Such systems, although allowing screening of large numbers of samples, are unsuited for studies that require cells with polar morphology. FTOC and NPOC studies established key events in GC and MC infections of mucosal epithelia. These ex vivo systems, composed of native tissue segments, made possible certain studies of neisserial infection such as ciliotoxicity, times of trafficking, and cytokine release. However, such systems require technical specialization and are 
limited by the increasing scarcity of appropriate tissue. The $\mathrm{T}_{84}$ system overcomes many of these difficulties. It will be interesting to use this model to dissect the cell biology of neisserial passage across epithelial barriers and to elucidate further the reciprocal roles of host and parasite in this important pathogenic process.

\section{ACKNOWLEDGMENTS}

We wish to thank Dr. J. L. Madara, C. Enns, J. Nelson, F. Heffron, and two anonymous reviewers for critical comments on this manuscript. This work was supported in part by National Institutes of Health Grant RO1-AI32493 to MS. DBR received a Howard Hughes predoctoral fellowship.

\section{REFERENCES}

1. Cohen MS, Sparling PF. (1992) Mucosal infection with Neisseria gonorrhoeae: Bacterial adaptation and mucosal defenses. J. Clin. Invest. 89: 1699-1705.

2. Nassif X, So M. (1995) Interaction of pathogenic Neisseriae with nonphagocytic cells. Clin. Microbiol. Rev. 8: 376-388.

3. Laga M, et al. (1993) Non-ulcerative sexually transmitted diseases as risk factors for HIV-1 transmission in women: Results from a cohort study. AIDS 7: 95-102.

4. Weir SS, Feldblum PJ, Roddy RE, Zekeng L. (1994) Gonorrhea as a risk factor for HIV acquisition. AIDS 8: 1605-1608.

5. McGee ZA, Stephens DS, Hoffman LH, Schlech WF, Horn RG. (1983) Mechanisms of mucosal invasion by pathogenic Neisseria. Rev. Infect. Dis. 5(Suppl 4): S708-S714.

6. Stephens DS, Farley MM. (1991) Pathogenic events during infection of the human nasopharynx with Neisseria meningitids and Haemophilus influenzae. Rev. Infect. Dis. 13: 22-33.

7. Silverstein SC, Steinman RM, Cohn ZA. (1977) Endocytosis. Annu. Rev. Biochem. 46: 669-722.

8. Shaw JH, Falkow S. (1988) Model for invasion of human tissue culture cells by Neisseria gonnorhoeae. Infect. Immun. 56: 1625-1632.

9. Chen JC-R, Bavoil P, Clark VL. (1991) Enhancement of the invasive ability of Neisseria gonorrhoeae by contact with HeclB, an adenocarcinoma endometrial cell line. Mol. Microbiol. 5: 1531-1538.

10. Cohen MS, Cannon JG, Jerse AE, Charniga LM, Isbey SF, Whicker LG. (1994) Human experimentation with Neisseria gonorrhoeae: Rationale, methods, and implications for the biology of infection and vaccine development. J. Infect. Dis. 169: 532-537.

11. Bessen D, Gotschlich EC. (1986) Interactions of gonococci with HeLa cells: Attachment, detachment, replication, penetration, and the role of protein II. Infect. Immun. 54: 154-160.

12. Weel JFL, Hopman CTP, van Putten JPM. (1991) In situ expression and localization of Neisseria gonorrhoeae opacity proteins in infected epithelial cells: Apparent role of Opa proteins in cellular invasion. J. Exp. Med. 173: 1395-1405.

13. van Putten JPM. (1993) Phase variation of lipopolysaccharide directs interconversion of invasive and immuno-resistant phenotypes of Neisseria gonorrhoeae. EMBO J. 12: 4043-4051.

14. Nassif X, Lowy J, Stenberg P, O'Gaora P, Ganji A, So M. (1993) Antigenic variation of pilin regulates adhesion of Neisseria meningitidis to human epithelial cells. Mol. Microbiol. 8: 719-725.

15. Virji M, Kayhty H, Ferguson DJP, Alexandrescu C, Heckels JE, Moxon ER. (1991) The role of pili in the interactions of pathogenic Neisseria with cultured human endothelial cells. Mol. Microbiol. 5: 1831-1841.

16. Virji M, Makepeace K, Moxon ER. (1994) Distinct mechanisms of interactions of Opcexpressing meningococci at apical and basolateral surfaces of human endothelial cells; The role of integrins in apical interactions. Mol. Microbiol. 14: 173-184.

17. Grobstein C. (1956) Trans-filter induction of tubules in mouse metanephrogenic mesenchyme. Exp. Cell Res. 10: 424-440.

18. Cereijdo M, Robbins ES, Dolan WJ, Rotunno CA, Sabatini DD. (1978) Polarized monolayers formed by epithelial cells on a permeable and translucent support. J. Cell. Biol. 77: 853-879.

19. Mostov KE, Deitcher DL. (1986) Polymeric immunoglobulin receptor expressed in MDCK cells transcytoses IgA. Cell 46: 613-621.

20. Rodriguez-Boulan EJ, Pendergrast M. (1980) Polarized distribution of viral envelope glycoprotein in the plasma membrane of infected epithelial cells. Cell 20: 45-54.

21. Dharmsathaphorn K, Madara JL. (1990) Established intestinal cell lines as model systems for electrolyte transport studies. Methods Enzymol. 192: 354-389.

22. Madara JL. (1988) Tight junction dynamics: 
Is paracellular transport regulated? Cell 53: 497-498.

23. Finlay BB, Gumbiner B, Falkow S. (1988) Penetration of Salmonella through a polarized Madin-Darby canine kidney epithelial cell monolayer. J. Cell Biol. 107: 221-230.

24. Finlay BB, Falkow S. (1990) Salmonella interactions with polarized human intestinal Caco-2 epithelial cells. J. Infect. Dis. 162: 1096-1 106.

25. Madara JL. (1994) Migration of neutrophils through epithelial monolayers. Trends Cell Biol. 4: 4.

26. McCormick BA, Hofman PM, Kim J, Carnes DK, Miller SI, Madara JL. (1995) Salmonella typhimurium attachment to human intestinal epithelial monolayers: Transcellular signaling to subepithelial neutrophils. J. Cell Biol. 131: 1599-1608.

27. Birkness KA, Swisher BL, White EH, Long EG, Ewing EP, Quinn FD. (1995) A tissue culture bilayer model to study the passage of Neisseria meningitidis. Infect. Immun. 63: 402-409.

28. Canil C, Rosenshine I, Ruschkowski S, Donnenberg MS, Kaper JB, Finlay BB. (1993) Enteropathogenic Escherichia coli decreases the transepithelial electrical resistance of polarized epithelial monolayers. Infect. Immun. 61: 2755-2762.

29. Jepson MA, Collares-Buzato CB, Clark MA, Hirst BH, Simmons NL. (1995) Rapid disruption of epithelial barrier function by Salmonella typhimurium is associated with structural modification of intercellular junctions. Infect. Immun. 63: 356-359.

30. Meyer TF, Billyard E, Haas R, Storzbach S, So M. (1984) Pilus sequences of Neisseria gonorrhoeae: Chromosomal organization and DNA sequence. Proc. Natl. Acad. Sci. U.S.A. 86: $5054-5058$.

31. Segal E, Billyard E, So $M$, Storzbach S, Meyer TF. (1985) Role of chromosomal rearrangement in $N$. gonorrhoeae pilus phase variation. Cell 40: 293-300.

32. Trieu-Cout P, Poyart-Salmeron C, Carlier C, Courvalin P. (1990) Nucleotide sequence of the erythromycin resistance gene of the conjugative transposon Tn1545. Nucleic Acids Res. 18: 3660.

33. Waldbeser LS, Ajioka RS, Merz AJ, Puaoi D,
Lin L, Thomas M, So M. (1994) The opaH locus of Neisseria gonorrhoeae MS1lA is involved in epithelial cell invasion. Mol. Microbiol. 13: 919-928.

33a.Sparling PF. 1966. Genetic transformation of Neisseria gonorrhoeae to streptomycin resistance. J. Bacteriol. 92: 1364-1371.

34. Rochat T, Casale J, Hunninghake GW, Peterson MW. (1988) Neutrophil cathepsin G increases permeability of cultured type II pneumocytes. Am. J. Physiol. 255: C603-C611.

35. Zamboni L, De Martino C. (1967) Buffered picric acid paraformaldehyde: A new, rapid fixative for electron microscopy. J. Cell Biol. 35: 148A.

36. Sokal RR, Rohlf FJ. (1981) Biometry. 2nd ed. W. H. Freeman and Company, New York.

37. Stephens DS, Hoffman LH, McGee ZA. (1983) Interaction of Neisseria meningitidis with human nasopharyngeal mucosa: Attachment and entry into columnar epithelial cells. J. Infect. Dis. 148: 369-376.

38. McGee ZA, Johnson AP, Taylor-Robinson D. (1981) Pathogenic mechanisms of Neisseria gonorrhoeae: Observations on damage to human Fallopian tubes in organ culture by gonococci of colony type 1 or type 4 . J. Infect. Dis. 143: 413-422.

39. Schmidt A, Heid HW, Schafer S, Nuber UA, Zimbelmann R, Franke W. (1994) Desmosomes and cytoskeletal architecture in epithelial differentiation: Cell type-specific plaque components and intermediate filament anchorage. Eur. J. Cell Biol. 65: 229-245.

40. Kellogg DS, Peacock WL, Deacon WE, Brown L, Prikle CI. (1963) Neisseria gonorrhoeae. I. Virulence genetically linked to clonal variation. J. Bacteriol. 85: 1274-1279.

41. Swanson J. (1988) Expression of outer membrane protein II by gonococci in experimental gonorrhea. J. Exp. Med. 168: 2121-2129.

42. Swanson J. (1973) Studies on gonococcus infection. IV. Pili: Their role in attachment of gonococci to tissue culture cells. J. Exp. Med. 137: $571-589$.

43. Makino S, van Putten JPM, Meyer TF. (1991) Phase variation of the opacity outer membrane protein controls invasion by Neisseria gonorrhoeae into human epithelial cells. EMBO J. 10: 1307-1315.

Contributed by S. Falkow. Accepted on August 8, 1996. 